\title{
Blind Principles Based Interference and Noise Reduction Schemes for OFDM
}

\author{
M. G. S. Sriyananda · J. Joutsensalo • T. Hämäläinen
}

Published online: 4 November 2012

(C) The Author(s) 2012. This article is published with open access at Springerlink.com

\begin{abstract}
One of the premier mechanisms used in extracting unobserved signals from observed mixtures in signal processing is employing a blind source separation (BSS) algorithm or technique. A prominent role in the sphere of multicarrier communication is played by orthogonal frequency division multiplexing (OFDM) techniques. A set of remedial solutions taken to mitigate deteriorative effects caused within the air interface of an OFDM transmission with aid of BSS schemes is presented. Two energy functions are used in deriving the filter coefficients. They are optimized and performance is justified. These functions with the iterative fixed point rule for receive signal are used in determining the filter coefficients. Time correlation properties of the channel are taken advantage for BSS. It is tried colored noise and interference components to be removed from the signal mixture at the receiver. The method is tested in a slow fading channel with a receiver containing equal gain combining to treat the channel state information values. The importance is that, these solutions can be noted as quite low computational complexity mechanisms.
\end{abstract}

Keywords Blind source separation · OFDM $\cdot$ Slow fading $\cdot$ Downlink

\section{Introduction}

Blind source or signal separation consists of recovering unobserved signals or sources from several observed mixtures. Typically the observation values are obtained at the output of a set of sensors, where each sensor receives a different combination of the source signals. The

M. G. S. Sriyananda $(\varangle)$ · J. Joutsensalo · T. Hämäläinen

Department of Mathematical Information Technology, Faculty of Information Technology,

P.O. Box 35, 40014, University of Jyväskylä, Finland

e-mail: gamage.m.s.sriyananda@jyu.fi

J. Joutsensalo

e-mail: jyrki.j.joutsensalo@jyu.fi

T. Hämäläinen

e-mail: timo.t.hamalainen@jyu.fi 
adjective "blind" stresses the two scenarios. First is that the source signals are not observed. Second is no information is available about the mixture. In other words, no prior information is available about the transfer. The lack of prior knowledge about the mixture is compensated by a statistically strong but often physically plausible assumption of independence between the source signals. The weakness of the prior information is precisely the strength of the BSS model, making it a versatile tool for exploiting the spatial diversity provided by an array of sensors. Since training or pilot data sequences are not required by blind schemes higher effective data rates are expected to be delivered. Statistical or structural properties of the transmitted signal are used by them. Semi-blind schemes are developed to overcome some of the drawbacks of blind schemes with reduced amount of training or pilot data. Principal components analysis, singular value decomposition, independent component analysis, dependent component analysis, non-negative matrix factorization, low-complexity coding and decoding and stationary subspace analysis can be introduced as the key categories or branches of BSS.

One of the main focuses is consideration of applicability of BSS principles to wireless communication systems. These BSS algorithms that are supported by energy criterion functions to be optimized. The performance of the function is justified in the manuscript. It is always advantageous to utilize these types of approaches to further strengthen the performance of well established most demanded systems based on the techniques like OFDM. That is a part of attempt in meeting the ever increasing demand for higher capacity communication systems. A set of most historical attempts of using BSS algorithms in communications, signal processing and neural networks can be highlighted as [1-3].

\section{BSS Models and Applications}

The basic BSS models and algorithms can be extended in several directions and more sensors than sources which are of much practical importance $[4,5]$. They include noisy observations and complex signals and mixtures. They are applicable to the standard narrow band array processing or beamforming models and convolutive mixtures leading to multichannel blind deconvolution problems. Some of these approaches can only separate stationary non-Gaussian signals. Due to these limitations, poor performance is obtained when dealing with some real sources, like audio signals. But there are different approaches which take advantage of the nonstationarity of sources in order to achieve better performance than the classical methods [6].

Biomedical signal processing and analysis (ECG, EEG, MEG, cancer prevention and diagnosis, modeling of the human body) [7-9], is an area that can be efficiently and effectively supported by BSS schemes where the driving force is a basic human need. Another highly demanded area is signal processing in communications including wireless communications [10] whose progress is propelled by ever growing demand for higher effective data rates and enhanced user mobility. Both can be included to the category of BSS based applications that are extensively connected with the day to day requirements of the society. Data mining, geophysical data processing, acoustics (audio signal processing), speech recognition, image recognition, electrical engineering [11], computer vision and machine learning are some themes which are been heavily researched where prominent role can be played by BSS algorithms. Lesser demanded applications also can be seen. But some of them are with great foreseen potential to fulfill important needs if they are been properly developed. In one such case, a problem of separating multiple speakers from mixtures of these that are obtained using multiple microphones in a room is addressed. An adaptive BSS algorithm entirely based on 
second order statistics which can run in offline or online [12] is given as the solution. A statistical analysis of microarray data using BSS theories for identifying "elementary" independent expression patterns is unveiled in [13]. In addition to all those applications, basic techniques and formulas that can be tested for specific applications are also widely available [14].

\subsection{General BSS Model}

When the output signals are independent, separation of the sources of linear instantaneous systems by combining the observations by means of a matrix adapted is aimed in [6]. The underlying assumption of these techniques is that the sources must be independent.

The basic BSS receive signal matrix model $\mathbf{x}(t), \mathbf{x}(t)=\left[x_{1}(t), x_{2}(t), \ldots, x_{f}(t), \ldots\right.$, $\left.x_{F}(t)\right]^{T}$ at time $t$ of a basic BSS model with $B$ unspecified inputs or sources and $F$ output or sensor observations has the form $[6,15,16]$,

$$
\mathbf{x}(t)=\mathbf{A s}(t)+\mathbf{w}(t)=s_{1}(t) \mathbf{a}_{1}+\mathbf{w}^{\prime}(t)
$$

where $\mathbf{A}$ is enclosed with mixture coefficient values and a $F \times B$ matrix. The element $f, b$ of it is given by a $f, b$. Source signal bearing $B$ sources $s_{1}(t), s_{2}(t), \ldots, s_{b}(t), \ldots, s_{B}(t)$ is signified by $\mathbf{s}(t) . \mathbf{a}_{1}, \mathbf{a}_{1}=\left[\mathrm{a}_{1,1}, \mathrm{a}_{2,1}, \ldots, \mathrm{a}_{f, 1}, \ldots, \mathrm{a}_{F, 1}\right]^{T}$ is the coefficient value matrix of $s_{1}(t) . s_{b}(t)$ is the signal element $b$ at time $t$. Components $s_{2}(t), s_{3}(t), \ldots, s_{b}(t), \ldots, s_{B}(t)$ are independent or uncorrelated symbols or signals. Signal $s_{1}(t)$ is time correlated. $\mathbf{w}(t)=\left[w_{1}(t), w_{2}(t), \ldots, w_{f}(t), \ldots, w_{F}(t)\right]^{T}$ is additive independent and identically distributed (IID) white noise and colored noise is given by $\mathbf{w}^{\prime}(t) . w_{f}(t)$ is the noise element $f$ at time $t$. When appropriately shaped mapped symbols are considered and the time delay is sufficiently shorter $c_{1}=\mathrm{E}\left\{s_{1}(t) s_{1}(t+\tau)\right\} \approx \mathrm{E}\left\{s_{1}^{2}(t)\right\}$. The differential correlation matrix $\mathbf{C}(\tau)$ at a small time difference $\tau$ is given [17] as follows,

$$
\mathbf{C}(\tau)=\mathrm{E}\left\{\mathbf{x}(t) \mathbf{x}^{T}(t+\tau)\right\}=c_{1} \mathbf{a}_{1} \mathbf{a}_{1}^{T}+\mathbf{R}_{\tau}
$$

where matrix transpose is denoted by ${ }^{T}$. The ordinary correlation matrix $\mathbf{R}$ is,

$$
\begin{aligned}
\mathbf{R} & =\mathrm{E}\left\{\mathbf{x}(t) \mathbf{x}^{T}(t)\right\} \\
& =\mathbf{A} \mathrm{E}\left\{\mathbf{s}(t) \mathbf{s}^{T}(t)\right\} \mathbf{A}^{T}+\sigma^{2} \mathbf{I} \\
& =c_{1} \mathbf{a}_{1} \mathbf{a}_{1}^{T}+\mathbf{R}_{0}
\end{aligned}
$$

Noise variance is given by $\sigma^{2}$. Assume that $\left\|\mathbf{R}_{0}\right\|>\left\|\mathbf{R}_{\tau}\right\|$, where $\|\cdot\|$ is Frobenius or 2-norm.

The output $\mathbf{y}(t)$ or $\mathbf{y}$ of the receiver with coefficient $\mathbf{u}_{r c}$ is given by,

$$
\mathbf{y}=\mathbf{u}_{r c}^{T} \mathbf{x}
$$

The output power $\mathrm{E}\left\{\mathbf{y}^{2}(t)\right\}$ can be derived by (3) and (4) as,

$$
\mathrm{E}\left\{\mathbf{y}^{2}(t)\right\}=\mathbf{u}_{r c}^{T} \mathbf{R} \mathbf{u}_{r c}
$$

A set of energy functions are considered under two categories, where the first category consisting $J_{1}(\mathbf{u})$ and $J_{2}(\mathbf{u})$ is for the removal of colored noise and the second category consisting $J_{3}(\mathbf{u})$ and $J_{4}(\mathbf{u})$ is for the removal of interference signal. $\mathbf{u}$ is a variable coefficient similar to $\mathbf{u}_{r c}$. Where the differential cross correlation matrix estimated from the Eq. (2), the Lagrangian multipliers and the matrix inverse are indicated by $\mathbf{C}, \lambda_{\beta}, \beta=1,2, \ldots, 4$ and ${ }^{-1}$ correspondingly. 


\subsubsection{For Colored Noise}

$$
\begin{aligned}
& J_{1}(\mathbf{u})=\mathbf{u}^{T} \mathbf{C u}+\lambda_{1}\left(\mathbf{I}-\mathbf{u}^{T} \mathbf{R}^{-1} \mathbf{u}\right) \\
& J_{2}(\mathbf{u})=\mathbf{u}^{T} \mathbf{C u}+\lambda_{2}\left(\mathbf{I}-\mathbf{u}^{T} \mathbf{R u}\right)
\end{aligned}
$$

Removal of the noise component of the interference $\mathbf{w}^{\prime}(t)$ is aimed while passing the signal $s_{1}(t) \mathbf{a}_{1}$. The energy function is a dependent of the measured signal values $\mathbf{x}(t)$ or $\mathbf{x}$. The first terms of (6) and (7) try to minimize the projection of $\mathbf{u}$ to the effective spaces spanned by the matrix $\mathbf{C}^{-1}$. The output power to that kind of inverse process is minimized by $\mathbf{u}$, which the correlation matrix $\mathbf{C}^{-1}$ is produced. Differential correlation matrix $\mathbf{C}$ contains just time correlated signal part $s_{1}(t) \mathbf{a}_{1}$. Resulting $\mathbf{u}^{T} \mathbf{C}^{-1} \mathbf{u}$ is in minimum due to usage of the inverse matrix. Therefore it is expected that the signal $s_{1}(t) \mathbf{a}_{1}$ is passed quite well by $\mathbf{u}_{1}$. The projections of $\mathbf{u}$ to the effective spaces spanned by the matrix $\mathbf{R}$ is tried to minimized by the second term. $\mathrm{E}\left\{\mathbf{y}^{2}(t)\right\}$ is also tried to be minimized. But without differential correlation, $\mathbf{R}$ contains much the interference part $\mathbf{R}_{0}$. Then the desired signal $s_{1}(t) \mathbf{a}_{1}$ is filtered well again by $\mathbf{u}$.

Finding answers for these scenarios, a number of solutions can be developed under different conditions. Different approaches to get rid of or lessen the effect of noise or interference components can be noticed. A pair of suchlike solutions where noise component is tried to be removed is described bellow.

\section{- Algorithm 1}

Considering the partial derivative $\frac{\partial J_{1}(\mathbf{u})}{\partial \mathbf{u}}=0$ of (6) at $\mathbf{u}_{1}$ for the optimization,

$$
\lambda_{1} \mathbf{u}_{1}=\mathbf{R C} \mathbf{u}_{1}
$$

Colored noise is filtered out.

Using $\lambda_{1} \mathbf{u}_{1}(t)=\mathbf{g}_{1}^{1}(t)$ and the iterative fixed point rule as follows,

$$
\begin{aligned}
\mathbf{g}_{1}^{1}(t) & =\mathbf{R C} \mathbf{u}_{1}(t) \\
\mathbf{u}_{1}^{1}(t) & =\frac{\mathbf{g}_{1}^{1}(t)}{\left\|\mathbf{g}_{1}^{1}(t)\right\|} \\
\mathbf{g}_{1}^{2}(t) & =\mathbf{R C} \mathbf{u}_{1}^{1}(t)
\end{aligned}
$$

- Algorithm 2

Considering the partial derivative $\frac{\partial J_{2}(\mathbf{u})}{\partial \mathbf{u}}=0$ of (7) at $\mathbf{u}_{2}$ for the optimization,

$$
\lambda_{2} \mathbf{u}_{2}=\mathbf{C R}^{-1} \mathbf{u}_{2}
$$

Using $\lambda_{2} \mathbf{u}_{2}(t)=\mathbf{g}_{2}^{1}(t)$ and the iterative fixed point rule as follows,

$$
\begin{aligned}
\mathbf{g}_{2}^{1}(t) & =\mathbf{C R}^{-1} \mathbf{u}_{2}(t) \\
\mathbf{u}_{2}^{1}(t) & =\frac{\mathbf{g}_{2}^{1}(t)}{\left\|\mathbf{g}_{2}^{1}(t)\right\|} \\
\mathbf{g}_{2}^{2}(t) & =\mathbf{C R}^{-1} \mathbf{u}_{2}^{1}(t)
\end{aligned}
$$




\subsubsection{For Interference Signal}

$$
\begin{aligned}
& J_{3}(\mathbf{u})=\mathbf{u}^{T} \mathbf{C}^{-1} \mathbf{u}+\lambda_{3}\left(\mathbf{I}-\mathbf{u}^{T} \mathbf{R}^{-1} \mathbf{u}\right) \\
& J_{4}(\mathbf{u})=\mathbf{u}^{T} \mathbf{C}^{-1} \mathbf{u}+\lambda_{4}\left(\mathbf{I}-\mathbf{u}^{T} \mathbf{R} \mathbf{u}\right)
\end{aligned}
$$

As the prime aspiration of the process, the interference component of the interference $\mathbf{w}^{\prime}(t)$ is targeted to be suppressed while passing the signal $s_{1}(t) \mathbf{a}_{1}$. Minimization of the projections of $\mathbf{u}$ to the effective space spanned by the matrix $\mathbf{C}^{-1}$ is tried by the first terms of the (16) and (17). The output power to suchlike inverse method is minimized by $\mathbf{u}$, witch the correlation matrix $\mathbf{C}^{-1}$ is produced. Differential correlation matrix $\mathbf{C}$ contains just time correlated signal part $s_{1}(t) \mathbf{a}_{1}$. Due to usage of the inverse matrix resulting $\mathbf{u}^{T} \mathbf{C}^{-1} \mathbf{u}$ is kept in minimum. Therefore it is expected to pass the signal $s_{1}(t) \mathbf{a}_{1}$ quite well by $\mathbf{u}$. Minimization of the projection of $\mathbf{u}$ to the effective space spanned by the matrix $\mathbf{R}$ is tried by the second term. So $\mathrm{E}\left\{\mathbf{y}^{2}(t)\right\}$ is tried to be minimized. But without differential correlation, $\mathbf{R}$ contains much the interference part $\mathbf{R}_{0}$. Then the desired signal $s_{1}(t) \mathbf{a}_{1}$ is filtered well again by $\mathbf{u}$.

- Algorithm 1

Considering the partial derivative $\frac{\partial J_{3}(\mathbf{u})}{\partial \mathbf{u}}=0$ of (16) at $\mathbf{u}_{3}$ for the optimization,

$$
\lambda \mathbf{u}_{3}=\mathbf{C}^{-1} \mathbf{R} \mathbf{u}_{3}
$$

Interference signal is filtered out.

Using $\lambda_{3} \mathbf{u}_{3}(t)=\mathbf{g}_{3}^{1}(t)$ and the iterative fixed point rule as follows,

$$
\begin{aligned}
& \mathbf{g}_{3}^{1}(t)=\mathbf{C}^{-1} \mathbf{R} \mathbf{u}_{3}(t) \\
& \mathbf{u}_{3}^{1}(t)=\frac{\mathbf{g}_{3}^{1}(t)}{\left\|\mathbf{g}_{3}^{1}(t)\right\|} \\
& \mathbf{g}_{3}^{2}(t)=\mathbf{C}^{-1} \mathbf{R} \mathbf{u}_{3}^{1}(t)
\end{aligned}
$$

- Algorithm 2

Considering the partial derivative $\frac{\partial J_{4}(\mathbf{u})}{\partial \mathbf{u}}=0$ of (17) at $\mathbf{u}_{4}$ for the optimization,

$$
\lambda_{4} \mathbf{u}_{4}=(\mathbf{C R})^{-1} \mathbf{u}_{4}
$$

Interference signal is filtered out.

Using $\lambda_{4} \mathbf{u}_{4}(t)=\mathbf{g}_{4}^{1}(t)$ and the iterative fixed point rule as follows,

$$
\begin{aligned}
\mathbf{g}_{4}^{1}(t) & =(\mathbf{C R})^{-1} \mathbf{u}_{4}(t) \\
\mathbf{u}_{4}^{1}(t) & =\frac{\mathbf{g}_{4}^{1}(t)}{\left\|\mathbf{g}_{4}^{1}(t)\right\|} \\
\mathbf{g}^{2}(t) & =(\mathbf{C R})^{-1} \mathbf{u}_{4}^{1}(t)
\end{aligned}
$$

For the iteration $m$ of any of $\mathbf{u}_{1}(t), \mathbf{u}_{2}(t), \mathbf{u}_{3}(t)$, or $\mathbf{u}_{4}(t)$,

$$
\mathbf{u}_{c}^{m}(t)=\frac{\mathbf{g}_{c}^{m}(t)}{\left\|\mathbf{g}_{c}^{m}(t)\right\|}
$$

where $\cdot_{1}, \cdot_{2}, \cdot_{3}$ or $\cdot_{4}$ is represented by $\cdot_{c}$. 


\subsection{BSS for OFDM}

One of the dominant inherent advantages of OFDM is robustness to frequency-selective fading. High spectral efficiency and multipath tolerance are some other precious features associated with this technique. Contributions of BSS algorithms in symbol recovery at the receiver are tested for OFDM. There is an apparent need of low complexity schemes to remove noise and Interference components of a synchronous downlink OFDM systems under the same conditions.

Basic OFDM $[18,19]$ consisting $N$ subcarriers $[N$-point inverse discrete Fourier transform (IDFT)/discrete Fourier transform (DFT)] is considered. Channel frequency response of the channel state information (CSI) of subcarrier $n$ during an OFDM symbol period $t$ is given by $H_{n}(t)$. Channel impulse response for the path $l$ of the channel tap $k$ of the multipath frequency-selective Rayleigh fading channel within the same duration is denoted by $h_{k, l}(t)$. Each tap is supported by $L$ independent paths.

$$
H_{n}(t)=\sum_{k=0}^{N-1} \sum_{l=0}^{L-1} h_{k, l}(t) e^{-j \frac{2 \pi k n}{N}}
$$

The receive signal $r_{n}(t)$ on subcarrier $n$ during $t$ can be expressed as in (28).

$$
r_{n}(t)=H_{n}(t) d_{n}(t)+\left(\frac{\sigma}{\sqrt{2}} v_{n}(t)\right)
$$

where $d_{n}(t)$ and $v_{n}(t)$ are transmit symbol and normalized additive white Gaussian noise of subcarrier $n$ for the period $t$ respectively. $\sigma$ is the standard deviation to the additive white Gaussian noise. Since slow fading is considered it can be assumed that for a given symbol frame path gain $H_{n}(t)=H_{n}$ and $h_{k, l}(t)=h_{k, l}$.

$$
r_{n}(t)=H_{n} d_{n}(t)+\left(\frac{\sigma}{\sqrt{2}} v_{n}(t)\right)
$$

$P$ receive signal samples are taken with small time shifts of $\tau$ within each information symbol duration. Hence the receive signal samples of subcarrier $n$ at time period containing symbol $d_{n}(t)$ can be expressed as $r_{n}(t), r_{n}(t+\tau), \ldots, r_{n}(t+(p-1) \tau), \ldots, r_{n}(t+(P-1) \tau)$. Row matrix containing these samples as the elements is represented by the notation $\mathbf{r}_{n}(t)$. These samples are equivalent to $x_{1}(t), x_{2}(t), \ldots, x_{f}(t), \ldots, x_{F}(t)$ in (1). Symbol sample of $d_{n}(t)$ and additive white Gaussian noise of sample $p$ of receive signal $r_{n}(t)$ are indicated by $d_{n}(t+(p-1) \tau)$ and $v_{n}(t+(p-1) \tau)$ correspondingly. Using (29), sample $p$ of receive signal $r_{n}(t)$ can be given as,

$$
r_{n}(t+(p-1) \tau)=H_{n} d_{n}(t+(p-1) \tau)+\left(\frac{\sigma}{\sqrt{2}} v_{n}(t+(p-1) \tau)\right)
$$

For any symbol sample on subcarrier $n$ within a time duration of a transmit information symbol $d_{n}(t), d_{n}(t+(p-1) \tau)=d_{n}(t)$.

$$
r_{n}(t+(p-1) \tau)=H_{n} d_{n}(t)+\left(\frac{\sigma}{\sqrt{2}} v_{n}(t+(p-1) \tau)\right)
$$

The differential correlation matrix $\mathbf{C}_{n}(\tau)$ at a small time difference $\tau$ and ordinary correlation matrix $\mathbf{R}_{n}$ for subcarrier $n$ according to (2) and (3) are,

$$
\begin{aligned}
\mathbf{C}_{n}(\tau) & =\mathrm{E}\left\{\mathbf{r}_{n}(t) \mathbf{r}_{n}^{*}(t+\tau)\right\} \\
\mathbf{R}_{n} & =\mathrm{E}\left\{\mathbf{r}_{n}(t) \mathbf{r}_{n}^{*}(t)\right\}
\end{aligned}
$$


Matrix complex conjugate transpose is denoted by $*^{*}$.

Iterative fixed point rule can be applied as same as in (9) [or (13), (19), (23)], where resultant $g_{c, n}^{1}(t)$ and $g_{c, n}^{m}(t)$ pairs of subcarrier $n$ of the four algorithms can be presented as follows.

\subsubsection{For Colored Noise}

- Algorithm 1

$$
\begin{aligned}
& g_{1, n}^{1}(t)=\mathbf{R}_{n} \mathbf{C}_{n} u_{1, n}(t) \\
& g_{1, n}^{m}(t)=\mathbf{R}_{n} \mathbf{C}_{n} u_{1, n}^{m-1}(t)
\end{aligned}
$$

- Algorithm 2

$$
\begin{aligned}
& g_{2, n}^{1}(t)=\mathbf{C}_{n} \mathbf{R}_{n}^{-1} u_{2, n}(t) \\
& g_{2, n}^{m}(t)=\mathbf{C}_{n} \mathbf{R}_{n}^{-1} u_{2, n}^{m-1}(t)
\end{aligned}
$$

\subsubsection{For Interference Signal}

- Algorithm 1

$$
\begin{aligned}
& g_{3, n}^{1}(t)=\mathbf{C}^{-1} \mathbf{R} u_{3, n}(t) \\
& g_{3, n}^{m}(t)=\mathbf{C}^{-1} \mathbf{R} u_{3, n}^{m-1}(t)
\end{aligned}
$$

- Algorithm 2

$$
\begin{aligned}
& g_{4, n}^{1}(t)=(\mathbf{C R})^{-1} u_{4, n}(t) \\
& g_{4, n}^{m}(t)=(\mathbf{C R})^{-1} u_{4, n}^{m-1}(t)
\end{aligned}
$$

Coefficient $u_{c, n}^{m}(t)$ for iteration $m$ for subcarrier $n$ of any of the solutions given for OFDM is stated as,

$$
u_{c, n}^{m}(t)=\frac{g_{c, n}^{m}(t)}{\left\|g_{c, n}^{m}(t)\right\|}
$$

Refined receive signal similar to (4) is,

$$
y_{n}(t+(p-1) \tau)=u_{c, n}^{m *}(t) r_{n}(t+(p-1) \tau)
$$

Signal after equal gain combining (EGC) can be expressed as,

$$
y_{n}(t)=\frac{u_{c, n}^{m *}(t)}{P} \frac{H_{n}^{*}}{\left|H_{n}\right|^{2}} \sum_{p=1}^{P} r_{n}(t+(p-1) \tau)
$$

The simplified matrix format output $\mathbf{y}(t)$ or $\mathbf{y}$ of the receiver with coefficients $\mathbf{u}$ for these OFDM based techniques is given as,

$$
\mathbf{y}_{O F D M}=\mathbf{u}_{O F D M}^{T} \mathbf{x}_{O F D M}
$$


where $\mathbf{u}_{O F D M}$ is a $N \times N$ diagonal matrix containing elements $u_{c, 1}^{m}(t), u_{c, 2}^{m}(t)$, $\ldots, u_{c, n}^{m}(t), \ldots, u_{c, N}^{m}(t)$ on the diagonal. $\mathbf{x}_{O F D M}$ is a column matrix. Element $n$ of it can be given by,

$$
x_{n}=\frac{1}{P} \frac{H_{n}^{*}}{\left|H_{n}\right|^{2}} \sum_{p=1}^{P} r_{n}(t+(p-1) \tau)
$$

\section{System Model}

The system model used for the discrete time base band simulations with single transmit and single receive antenna is given in Fig. 1. The system is equipped with $N$ subcarrier OFDM transmitter $[18,19]$ which is operated under slow fading frequency-selective Rayleigh channel containing Rayleigh distributed amplitude and uniformly distributed phase values. It is assumed that transmit and receiver setup is properly synchronized under all the conditions. Iterative BSS based lesser complex algorithms are employed with multiple sampling for each receive symbol at the receiver. Among various combining techniques, stable conventional combining technique viz. EGC is used. It is assumed that perfect path gain values are available at the receiver. Same system model and parameters are used under the same channel conditions in order to test and verify the results of standard OFDM system [18,19] without multiple sampling mechanism for the receive symbols.

\section{System Parameters and Simulation Results}

Simulations are carried out for systems considering 64 subcarrier basic OFDM transmitters. Binary information bits are mapped with 4-Quadrature Amplitude Modulation (4-QAM). After interleaving they are serial to parallel converted among subcarriers and transmitted through slow fading channel. Signal samples are obtained corresponding to each of the receive symbol on every subcarrier that are to be used for the BSS solutions. Considering the properties of the channel [20] and time correlation properties of the binary wave forms [17], it is decided to take samples only within the first $20 \%$ of time duration of an information symbol. Each channel tap is modeled using 4 independent paths. It is assumed that there is no variation of the signal due to any other reason during a symbol duration. The assumption becomes stronger, since the system is operated under slow fading channel conditions. Systems with the BSS schemes are configured to operate a number of iterations using the fixed point rule. Only one receive antenna is used to capture signal samples.

\subsection{Performance with Different Number of Subcarriers}

\subsubsection{For Colored Noise}

The OFDM systems namely the system with two noise removal BSS algorithms and the standard OFDM system with different number of subcarriers are tested and where the results are presented in Fig. 2. All the other parameters and conditions are maintained as the same. The results of the BSS schemes worked out for 16, 32 and 64 subcarriers employing algorithms 1 and 2 are given by Fig. 2a,b accordingly. Receive signal sampling is limited to 15 samples for each of the receive symbol on every subcarrier. In this scenario the OFDM systems with 


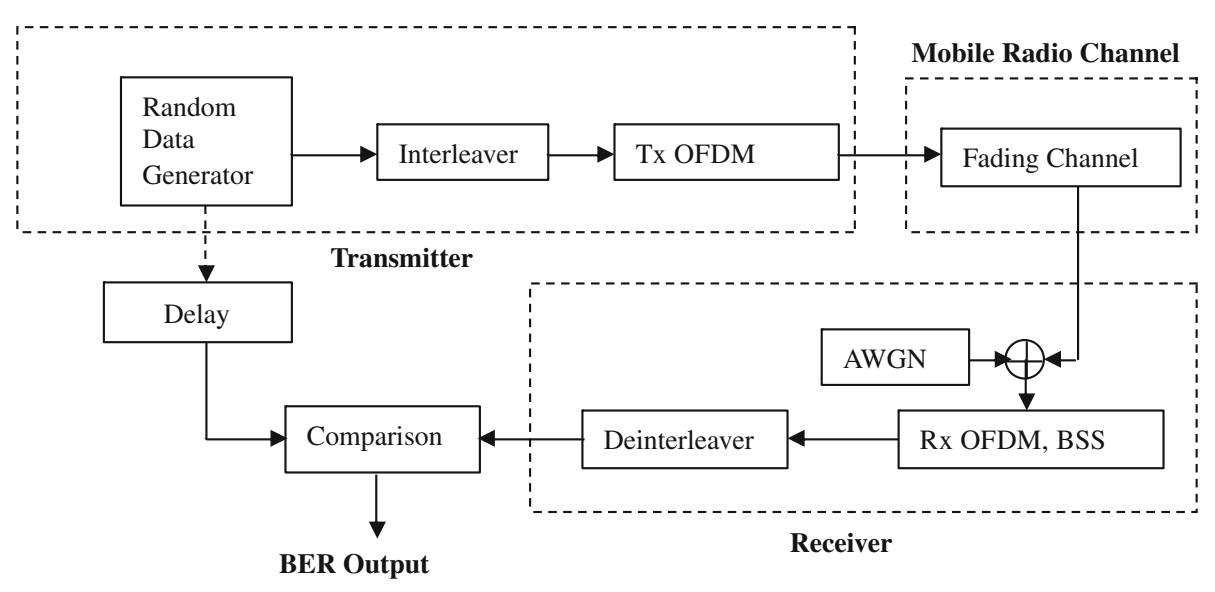

Fig. 1 Block diagram of the lowpass equivalent system model
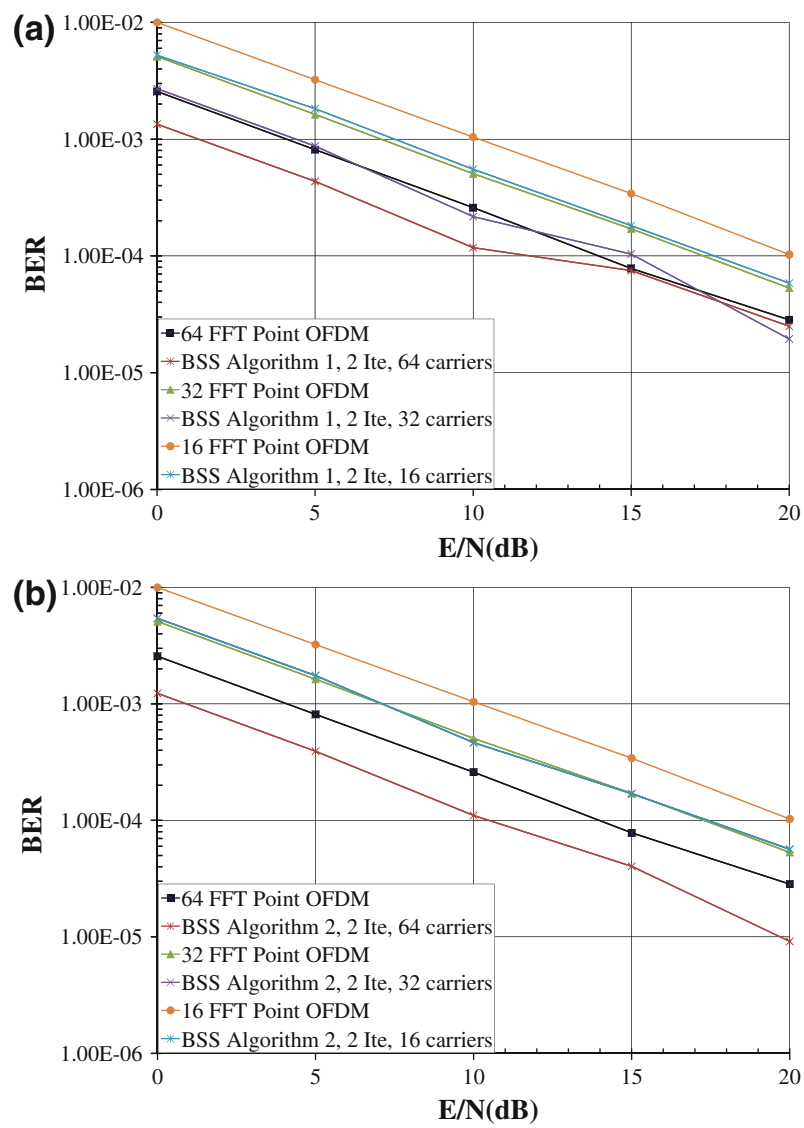

Fig. 2 OFDM systems with BSS and EGC : a noise removal Algorithm 1 and b noise removal Algorithm 2 
BSS algorithms are capable of outperforming the corresponding standard OFDM system. Sensitivity to noise can bee seen. Improvement in performance can be seen with the increase of signal power. That the iterative fixed point rule contributes negatively for the process of recovery of corrupted signals under these parameters.

\subsubsection{For Interference Signal}

In favor of the first set of simulations, variable number of subcarriers for the BSS scheme is considered. Performance results for 16, 32 and 64 subcarriers are obtained for this classification with 15 receive signal samples for each of the receive symbol on every subcarrier. Rest of the parameters and conditions of the model are maintained as the same. Outcome of the operations of the systems namely the OFDM system with the BSS algorithm and the standard OFDM system is presented in Fig. 3. The results of the scheme 1 and 2 worked out for 16, 32 and 64 subcarriers are given by Fig. 3a,b respectively. In this case the algorithms based OFDM systems are competent of outperforming the corresponding standard OFDM systems. But the iterative fixed point rule contributes destructively for the process of recovery
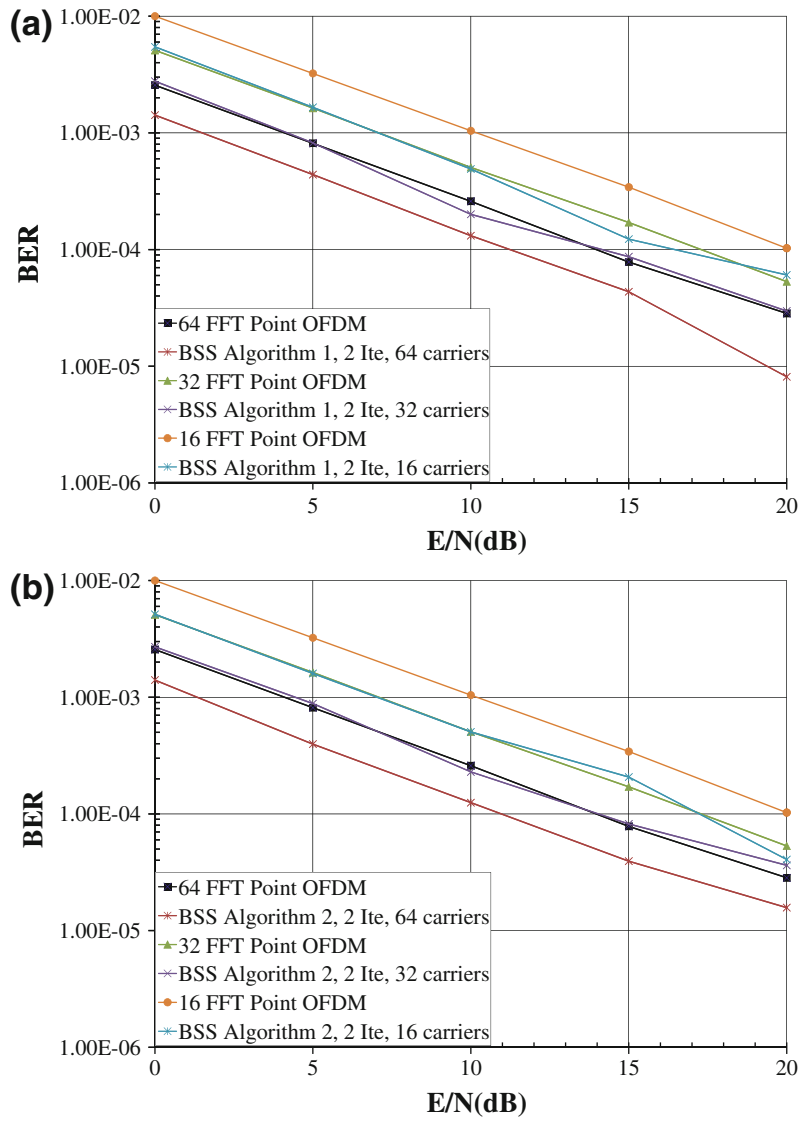

Fig. 3 OFDM systems with BSS and EGC : a interference signal removal Algorithm 1 and b interference signal removal Algorithm 2 

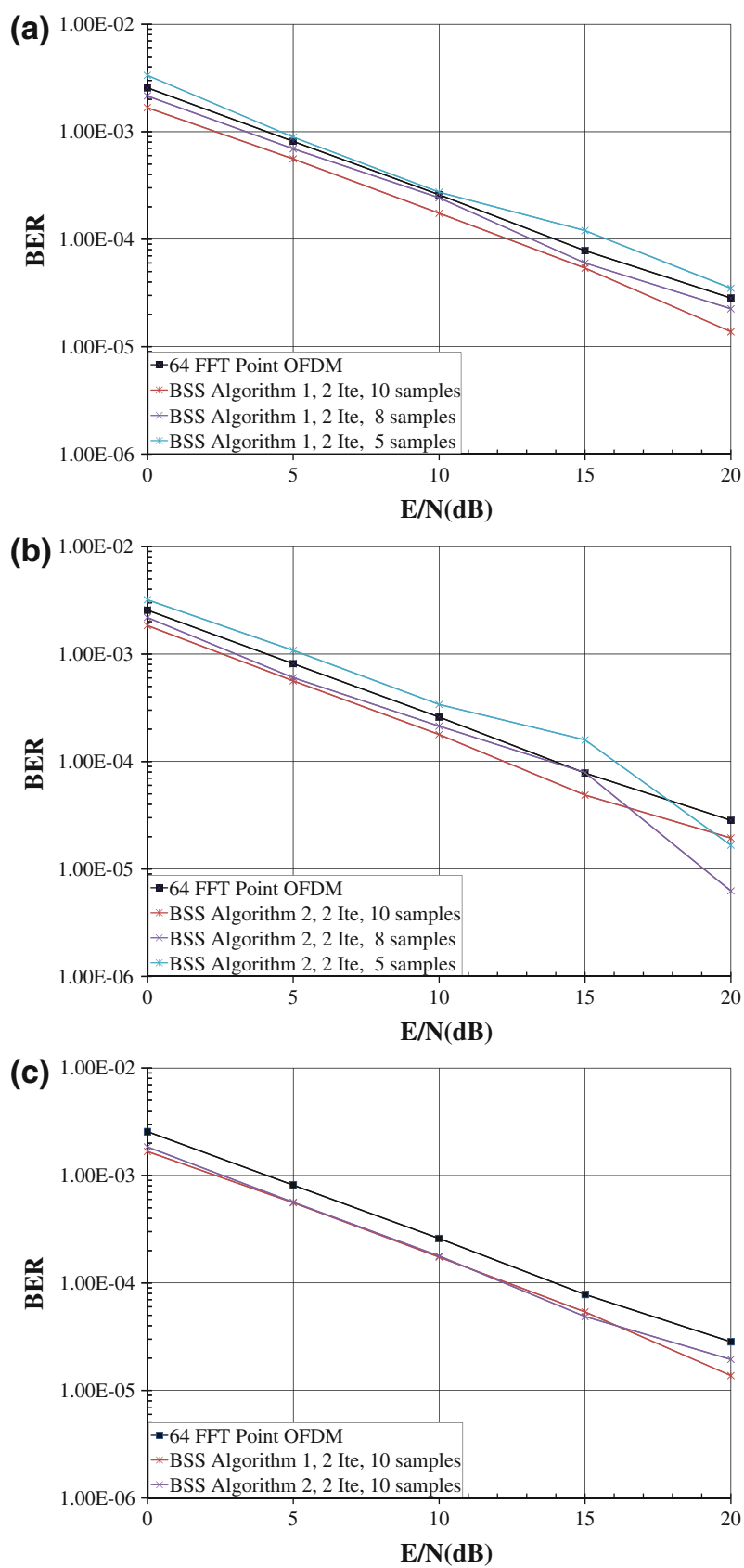

Fig. 4 OFDM systems with BSS and EGC : a noise removal Algorithm 1, b noise removal Algorithm 2 and c two algorithms with 10 samples

of corrupted signals under these parameters. Gradual improvement in performance with the increase of signal power can be seen. Behavior of the curves area in parallel to the curves of standard OFDM. 

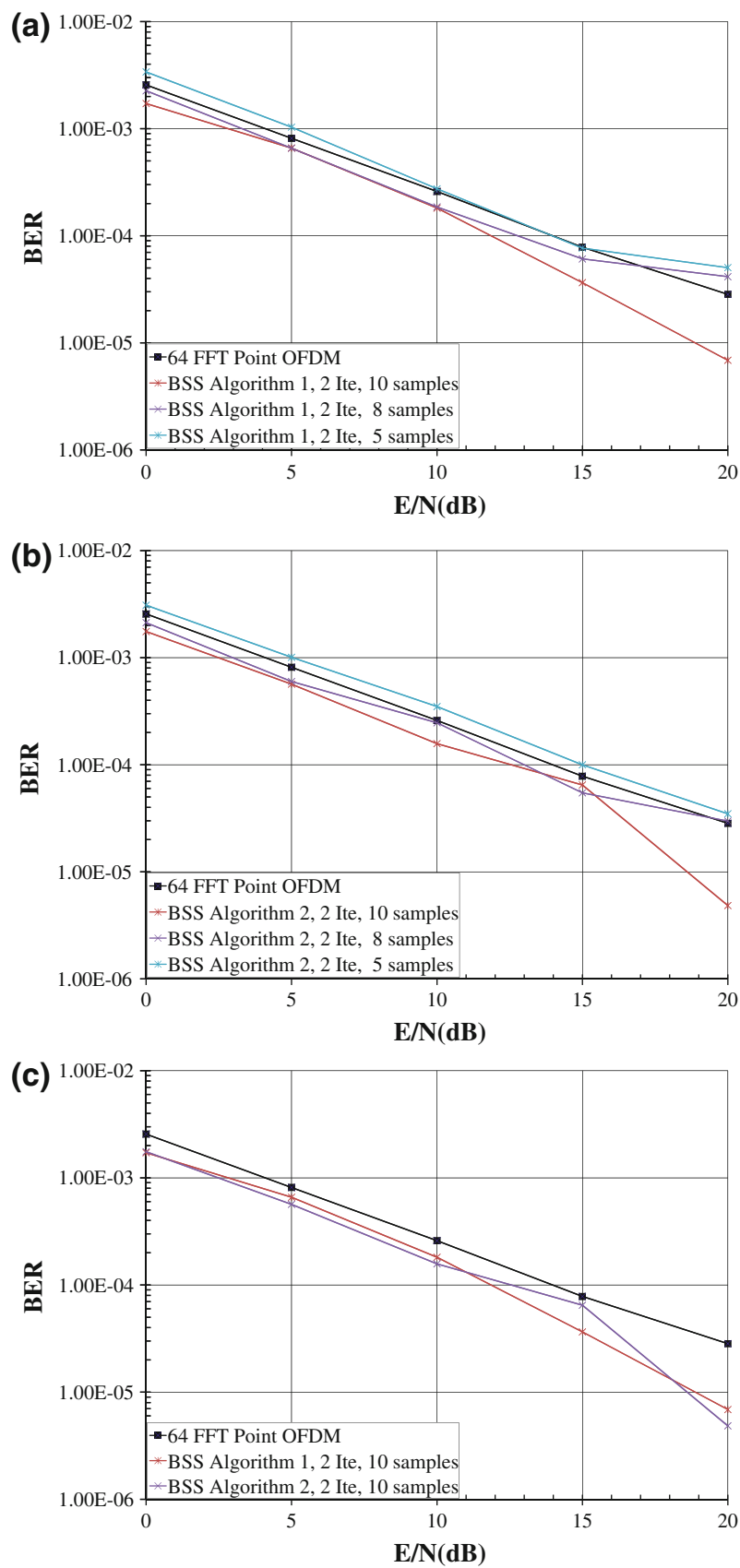

Fig. 5 OFDM systems with BSS and EGC : a interference signal removal Algorithm 1, b interference signal removal Algorithm 2 and $\mathbf{c}$ two algorithms with 10 samples 
4.2 Performance with Different Receive Signal Sample Sizes

\subsubsection{For Colored Noise}

Results of the simulations done for OFDM receiver with two BSS algorithms with different number of receive samples and standard OFDM system is presented by Fig. 4. Other parameters and conditions of the models are held as the same. Performance of the BSS schemes carried out taking 5, 8 and 10 receive signal samples for the two algorithms are shown by Fig. 4a,b respectively. Results of the both algorithms with 10 samples are presented in Fig. 4c. The simulation curves of the two schemes together with the standard or the normal OFDM system fall closer to each other. It can be observed that all these algorithms are capable of outperforming the standard OFDM system when higher numbers of samples are taken. With the increase of number of samples sizes of the matrices used for processing are also increased. Increasing the number of samples contributes positively for the process of refinery of corrupted symbols under these parameters.

\subsubsection{For Interference Signal}

Corroborative simulations conducted using the explained OFDM substructure with the BSS algorithm with different number of receive samples and standard OFDM system are shown in Fig. 5. Other parameters and conditions are maintained as the same. Performance is scrutinized for the BSS schemes carried out taking 5, 8 and 10 receive signal samples for the each algorithm are presented in Fig. 5a,b respectively. Outcome of the both algorithms with 10 samples is presented in $5 \mathrm{c}$ where the simulation curves of the two schemes fall in the vicinity of each other together with the standard OFDM system. Better bit error rates are demonstrated by the curves of taken for higher signal samples. In this scenario also with the increase of number of samples sizes of the matrices used for processing are also increased. Hence it could be resolved that a constructive contribution is done by a higher number of samples for the process of refinery of corrupted symbols under these parameters.

\section{Conclusion}

Better recovery of information symbols or an improvement in signal detection for the symbols transmitted in OFDM systems using four BSS solutions was considered. The for algorithms were derived, explained and tested with simulations comparing with a standard OFDM system. Reduction of destructive influence caused within free space communication was targeted specifically focusing on to removal of colored noise component and the removal of interference signal of receive signal. It is suggested to carry out more analysis for these algorithms covering much more realistic scenarios like Doppler effect before they are used in real environments.

Open Access This article is distributed under the terms of the Creative Commons Attribution License which permits any use, distribution, and reproduction in any medium, provided the original author(s) and the source are credited. 


\section{References}

1. Bar-Ness, Y., Carlin, J., \& Steinberger, M. (1982). Bootstrapping adaptive interference cancelers: Some practical limitations. IEEE Globecom Conference, pp. 1251-1255.

2. Hrault, J., Jutten, C., \& Ans, B. (1985). Dtection de grandeurs primitives dans un message composite par une architecture de calcul neuromimtique en apprentissage non supervis. In Actes du Xme colloque GRETSI, Nice, France, pp. 1017-1022.

3. Ans, B., Hrault, J., \& Jutten, C. (1985). Adaptive neural architectures: Detection of primitives. In Proceedings of COGNITIVA85, Paris, France, pp. 593-597.

4. Cardoso, J.-F. (1998). Blind signal separation: Statistical principles. Proceedings of the IEEE, 86(10), 2009-2025.

5. Sahin, M. E., Guvenc, I., \& Arslan, H. (2010). Uplink user signal separation for OFDMA-based cognitive radios. EURASIP Journal on Advances in Signal Processing, Hindawi Publishing Corporation, vol. 2010, Article ID 502369.

6. Abrard, F., \& Deville, Y. (2005). A timefrequency blind signal separation method applicable to underdetermined mixtures of dependent sources. Elsevier Journal on Signal Processing, 85, 1389-1403.

7. Ye, C., Kumar, B. V. K. V., \& Coimbra, M. T. (2012). Heartbeat classification using morphological and dynamic features of ECG signals. IEEE Transaction on Biomedical Engineering, 59(10), 2930-2941.

8. Haufe, S., Tomioka, R., Nolte, G., Müller, K.-R., \& Kawanabe, M. (2010). Modeling sparse connectivity between underlying brain sources for EEG/MEG. IEEE Transaction on Biomedical Engineering, 57(8), 1954-1963.

9. Zheng, C.-H., Huang, D.-S., Zhang, L., \& Kong, X.-Z. (2009). Tumor clustering using nonnegative matrix factorization with gene selection. IEEE Transactions on Information Technology in Biomedicine, 13(4), 599-607.

10. Waheed, M. E. (2009). Blind signal separation using an adaptive weibull distribution. International Journal of Physical Sciences, 4(5), 265-270.

11. Vizireanu, D. N. (2012). A fast, simple and accurate time-varying frequency estimation method for single-phase electric power systems. Measurement, 45(5), 1331-1333.

12. Schobben, D. W. E., \& Sommen, P. C. W. (1998). A new blind signal separation algorithm based on second order statistics. In Proceeding of IASTED international conference on signal and image processing, Las Vegas, USA.

13. Chiappetta, P., Roubaud, M. C., \& Torrésani, B. (2004). Blind source separation and the analysis of microarray data. Journal of Computational Biology, 11, 1090-1109.

14. Vizireanu, D. N., \& Halunga, S. V. (2012). Analytical formula for three points sinusoidal signals amplitude estimation errors. International Journal of Electronics, 99(1), 149-151.

15. Belouchrani, A., Abed-Meraim, K., Cardoso, J.-F., \& Moulines, E. (1997). A blind source separation technique using second-order statistics. IEEE Transactions on Signal Processing, 45(2), 434-444.

16. Parra, L., \& Sajda, P. (2003). Blind source separation via generalized eigenvalue decomposition. The Journal of Machine Learning Research, 4, 1261-1269.

17. Haykin, S. (2001). Communication systems (4th ed.). London: Wiley.

18. Sung, C. K., \& Lee, I. (2005). Multiuser bit-interleaved coded OFDM with limited feedback information. IEEE Vehicular Technology Conference, Dallas, Texas, USA.

19. Hanzo, L., Yang, L.-L., Kuan, E.-L., \& Yen, K. (2003). Single- and multi-carrier DSCDMA: Multi-user detection, space-time spreading, synchronisation, networking and standards. London: Wiley.

20. Proakis, J. G. (2001). Digital communications (4th ed.). New York: McGraw-Hill. 


\section{Author Biographies}
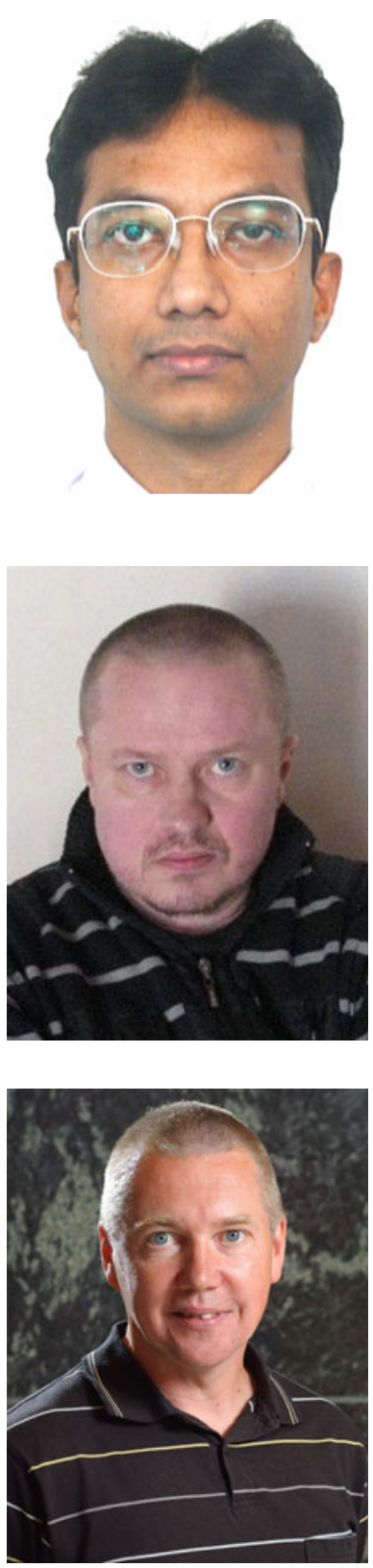

M. G. S. Sriyananda received the B.Sc. and M.Sc. degrees in Electronic and Telecommunication Engineering from University of Moratuwa, Sri Lanka. Currently, he is a doctoral student at Department of Mathematical Information Technology, University of Jyväskylä, Finland. $\mathrm{He}$ is a member of the university research and standardization project team related to air interface communication technologies. His research interests include wireless communication related channel coding and decoding, MIMO channel concepts, multiuser detection, multiple access technologies (single and multi user receiver technologies), and multicarrier techniques.

J. Joutsensalo was born in Kiukainen, Finland, in July 1966. He received diploma engineer, licentiate of technology, and doctor of technology degrees from Helsinki University of Technology, Espoo, Finland, in 1992, 1993, 1994, respectively. Currently, he is Professor of Telecommunications at the University of Jyväskylä. His research interests include signal processing for telecommunications, as well as data networks and pricing.

T. Hämäläinen received the B.Sc. degree in electrical engineering (1991) from the University of Applied sciences Jyvaskyla, the M.Sc degree in telecommunication (1996) from the Technical University of Tampere and the Ph.D. degree in telecommunication (2002), from the University of Jyvaskyla, Finland. Currently he is a Professor of Telecommunications at the University of Jyväskylä. His current research interests include performance evaluation and management of telecommunication networks and in particular mobility management, wireless communications, and network security. He is an editorial board member of journal WINET. 